International Journal of Engineering \& Technology, $7(2.8)(2018) 697-701$
International Journal of Engineering \& Technology
SPC
Website: www.sciencepubco.com/index.php/IJET
Research Paper

\title{
Priority based clustering the service providers in federated cloud
}

\author{
G S Mayuri ${ }^{1 *}$, M Saravanan ${ }^{2}$ \\ ${ }^{I}$ M.TECH, Cloud Computing, SRM Institute of Science and Technology, Kattankulathur. \\ ${ }^{2}$ Assistant professor, SRM Institute of Science and Technology, Kattankulathur \\ *Corresponding Author Email: mailtomayuricc@gmail.com
}

\begin{abstract}
Cloud is a trending technology now a days. In this technology world now everyone are moving with the cloud. A cloud federation technique is one of the best ways to select any services from the service providers by the user at any time. As large number of service providers in the federation, user need to be select best service provider and the selection of service provider get delayed in the federation. We proposed the cluster based approach to select the best service provider for user request and reducing the selection time of service provider. In previous papers there is no Automated Selection for the selection of best service provider. Our proposed work is based on Automated Selection model i.e., we applied k-means algorithm for grouping the similar services and categorizing the service providers into three groups. Such as Cluster-1, Cluster -2 and Cluster -3 , after cluster these cluster sorted in order based on the final centroid of each cluster. Now user request to be compared with the middle cluster centroid and directed to respective cluster for service selection. If more than one service provider offers similar services in the cluster then priority decision tree to be invoked for tie break. This technique works better than existing algorithms and time also to be saved in selection of service.
\end{abstract}

Keywords: K-Means, Priority Decision Tree, Categorization.

\section{Introduction}

The cloud is having terms, attributes, and administrations related to web-based registering, so it has alluded as distributed computing. The cloud is having a few Characteristics, for example, framework, provisioning, organize get to, and regulated metering are shown. The business advantage models being sent, (for instance, programming, stage, and establishment as an organization) and typical sending models used by master centers and customers to use and keep up the cloud organizations, (for instance, the private, open, gathering, and hybrid fogs) are discussed. We are having a few advantages and difficulties in distributed computing, and we are utilizing distributed computing administrations in various ways.

"Distributed computing" was instituted for what happens when applications and administrations are moved into the web "cloud." The cloud is only one access point from one point we can have $\mathrm{N}$ number of servers. Many organizations are conveying administrations from the cloud. Distributed computing isn't the simple thing which came at one overnight. The individuals endured such a great amount to execute this model

\section{Characteristics}

Cloud computing has a variety of characteristics, with the main ones being:

1) On-demand self-service: Punter can rarely plan dispensation abilities, for example, server period and system stockpiling as required unsurprisingly deprived of requiring human communication with specialist cooperative
2) Broad network access: Capabilities are reachable over system and got to through standard component that advances use by varied their or thick client programs and also other conservative or cloud-based software design administrations

3) Resource pooling: Gives figuring assets are pooled to serve numerous customers utilizing a multi-inhabitant demonstrate, with various physical and virtual assets progressively appointed and reassigned by purchaser request

4) Rapid elasticity: Abilities can be quickly and flexibly provisioned, naturally scaled out and quickly discharged to rapidly scale in. Capabilities accessible for provisioning seem, by all accounts, to be boundless and bought in any amount whenever 5) Measured services: Cloud structures thusly control and streamline resource use by using metering capacity at some level of reflection appropriate to the sort of organization. Resource utilize can be checked, controlled, uncovered. Giving straightforwardness to the supplier and buyer benefit so put it plainly, distributed computing takes into consideration the sharing and versatile arrangement of administrations, as required, from any area, and for which the client can be charged in light of genuine use.

\section{Related Work}

In the previous papers the ranking of services has done through the SLA's.in previous we have used Grade distribution algorithm and grade distribution table for ranking the best services.

It only support web services discovered in light of the utilitarian parts of administrations. In this choosing a reliable cloud service provider is a challenging issue in cloud environment. 
C. S. Rajarajeswari, M. Aramudhan, [1] (2014) Ranking Model for SLA Resource Provisioning Management. Displayed work gives another united cloud instrument, in which Cloud Broker Manager (CBM) takes up the responsibility of resource provisioning and ranking. Positioning the specialist organizations in view of cost and various parameters of cloud services like response time, security,etc.... he early universal Portrayal, Discovery, and Integration(UDDI) registries just help web administrations revelation in view of the utilitarian parts of administrations.

Preeti Gulia, Sumedha Sood Dynamic Ranking, and Selection of Cloud Providers Using Service Level Agreements [6]2013. Ranking the services selction of cloud providers through service level agreement(SLA's). Clients are confounding to pick the best and cost lessening administration, suppliers

Ahmad et al Dynamic Ranking and Selection of cloud suppliers utilizing Service Level Agreements 2015 the trust display in light of models of suitable administration quality and speed of execution for cloud assets. there are numerous testing issues, for example, security, encryption of information put away in the cloud and absence of trust in suppliers. Picking a solid specialist co-op is a testing issue in cloud condition.

$\mathrm{M}$

Aramudhan, M. Saravanan Trust-Based Ranking Service Models in Cloud Computing Environment [3]2016 web administrations are positioned in view of the QOS factors and by their favored cost along these lines influencing the clients to pick administrations as indicated by their advantage. web administrations being made accessible on the Internet, there is a should have the capacity to recognize them utilizing an arrangement of all around characterized Quality of Service (QoS) criteria.

\section{Proposed System}

Our proposed work is based on Automated Selection model i.e. we applied k-means algorithm for grouping the similar services and categorizing the service providers into three groups. Such as Cluster-1, Cluster -2 and Cluster -3 , after cluster these cluster sorted in order based on the final centroid of each cluster. Now user request to be compared with the middle cluster centroid and the request greater than the middle centroid value, then it to be directed to cluster -1 . If lesser than the middle cluster centroid, then it to be directed to cluster-3. At last if approximately equal to middle cluster centroid, then request to be processed by cluster 2. If more than one service provider offers similar services in the cluster then priority decision tree to be invoked for selection of best among the similar group. In this work consider centroid as user requirements and we will take the nearest values which satisfies the user requirements. All these ranked services are provided through federated cloud. Service models in cloud categorized as follows Infrastructure as a service (like Amazon/ Rackspace), SaaS, platform as service PaaS (like SalesForce.Com, Windows Azure).

In this we are proposing the clustering mechanism to find out the services easily. We can rank the services according to the user requirements. We are ranking to the services which are of software as a service, platform as a service, infrastructure as a service to all these services which are providing cloud services we will rank the best service provider to the user without any difficulty.

In this proposed system we are going to rank the services based on weightages given we can allot the weightages to the services as cluster 1 , cluster 2 , cluster 3 . This technique is used to increase the response time.it will provide the response to the user very fast within a time limit. we are going to the priority decision tree also to prioritize the best service among the cloud services

\section{A. Architecture}

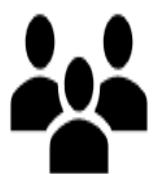

users
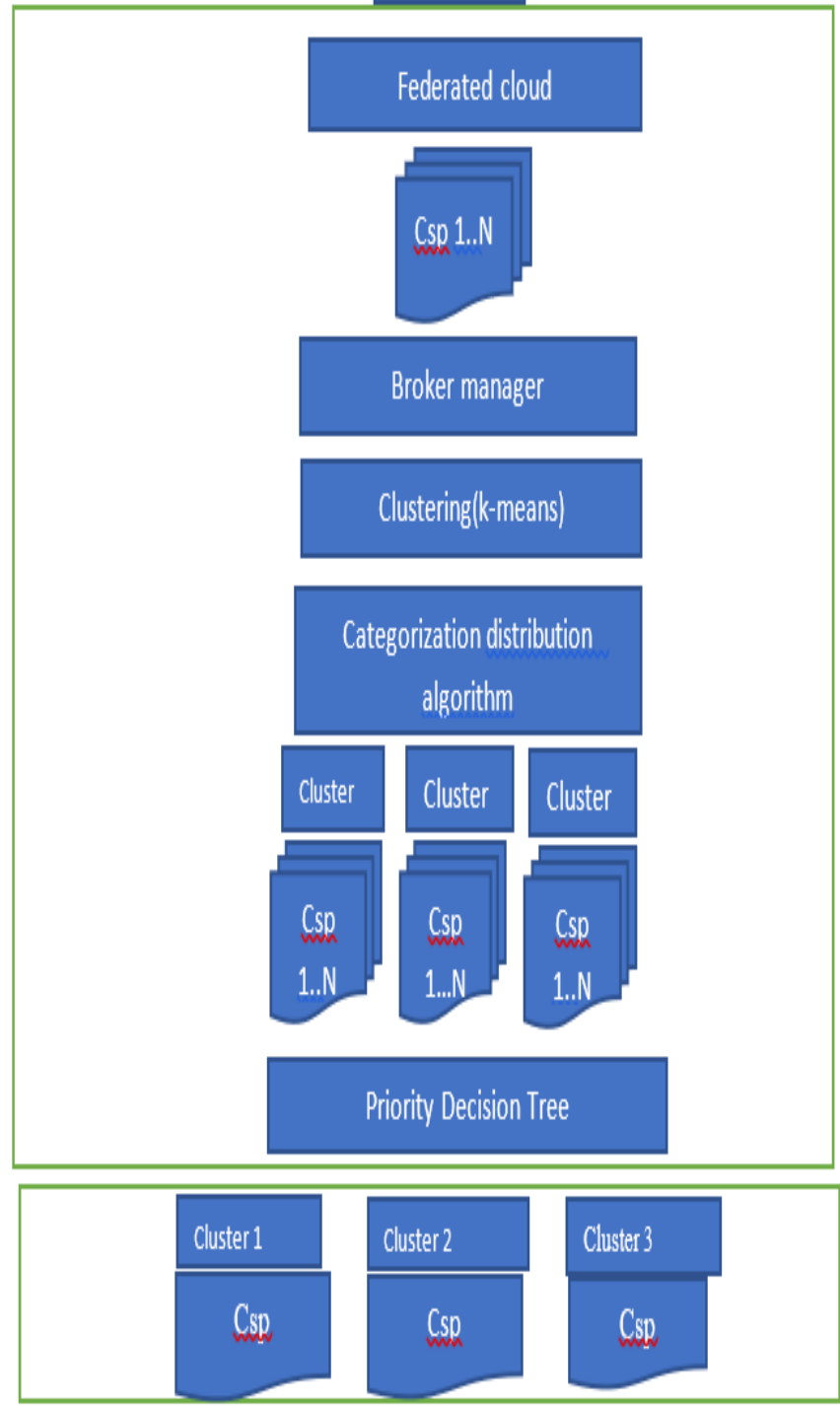

Fig. 1: Federated Architecture

In this federated architecture we are having the user, federated cloud service providers ,broker manager ,clustering mechanism ,categorization algorithm ,priority decision tree ,clouds service providers. So first the user will give the request for the best services he wants. It will pass the service request through the federates cloud

In the federated cloud all the actions will perform. It will perform the clustering technique for the cloud service providers the user request will be the centroid for clustering the technique after the clustering we will perform the categorization process it will categorize the service in to three categories they are cluster 1 ,cluster 2 ,cluster 3 then we will perform the priority decision tree for the cloud service providers 


\section{B. Selection procedure}

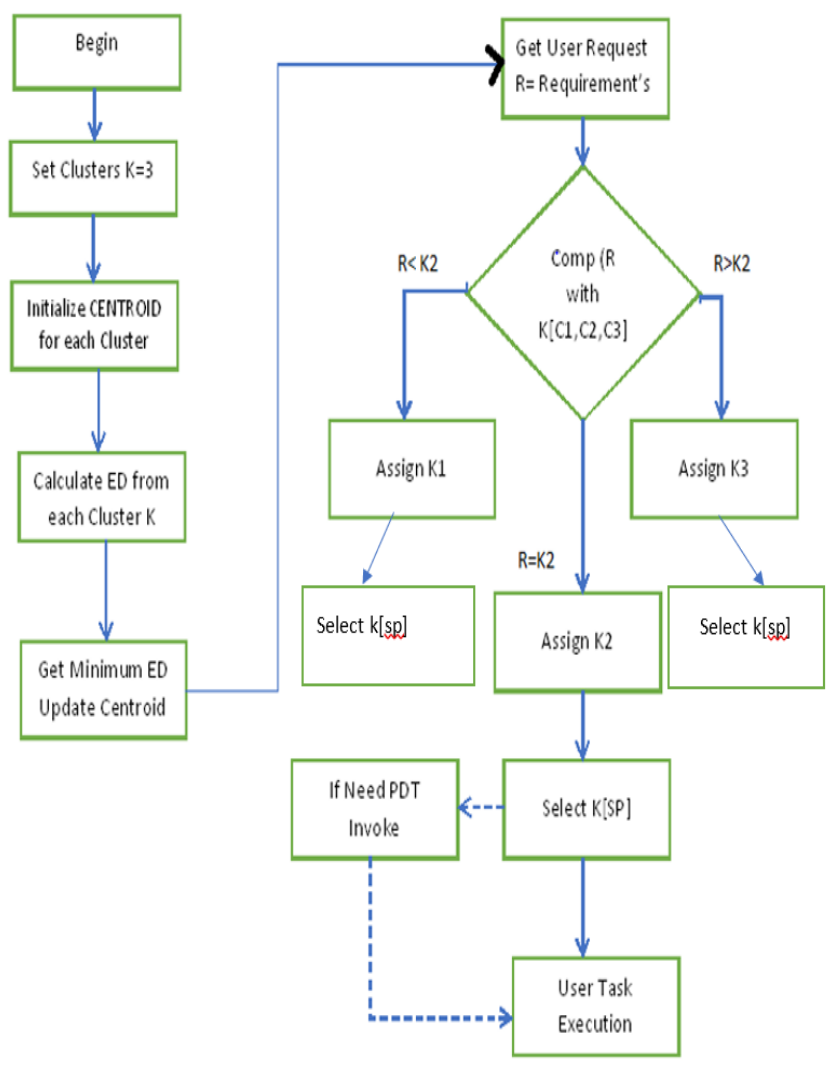

Fig. 2: Procedure for Cluster

\section{Algorithm}

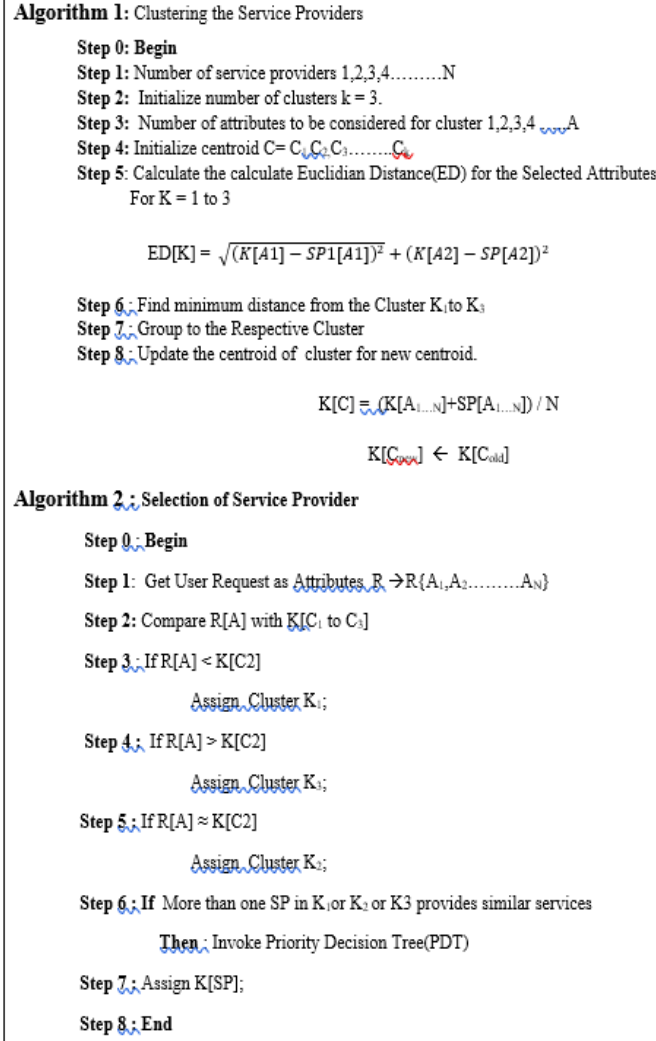

\section{Clustering Technique}

Bunching is worried about gathering the comparable protests together in a bunch and gathering the divergent questions in an alternate group. The focal point of a bunch is known as centroid might be a piece of the group or a nonexistent point. Grouping has far reaching applications, for example, in information mining, design acknowledgment, pressure and machine learning. The bunching calculation utilized as a part of this investigation is $\mathrm{K}$ implies calculation.

K-Means calculation parts the quantity of perception into ' $\mathrm{K}$ groups where each perception has a place with the bunch with the closest mean. This calculation is "NP-Hard to enhance", however the proficient heuristic calculation can be connected to merge rapidly to the nearby ideal. The quantity of groups ought to be predetermined to apply this calculation, i.e. express the estimation of $\mathrm{k}$. The execution of this calculation to a great extent relies upon the estimation of $\mathrm{K}^{\prime}$, it ought to be picked to such an extent that it mirrors a few attributes of the informational index under evaluation. Keeping in mind the end goal to productively pick the estimation of ' $K$ ', outline measure is utilized. The estimation of ' $K$ ' with most noteworthy normal outline width is decided for the informational collection

1 Firstly, the core of any information mining venture is the accumulation of information. In this task, the information for a segment of cloud service provider understudy of the services was considered.

2 Collected information was pre-prepared and the missing esteems are evacuated.

3 Data were standardized and reasonable weights were allocated to applicable traits of the information

4 The number of bunches that are required in k-implies grouping calculation is figured by taking the most extreme of outline measure among every one of the estimations of ' $k$ '.

$5 \mathrm{~K}$-implies calculation is connected on the pre-prepared informational index for getting the group.

This architecture shows that where clustering is going to perform and the federated architecture will be performing all the actions it cluster the dataset it is having with it and after clustering it will categorize the best service provider which user is seeing for and then priority decision is performed to rank the services as cluster 1 ,cluster 2 ,cluster

\section{Experiment Result}

We collected various data set of Service Providers(SP) and various attributes such as AWS- amazon web services ,Windows Azure, Google cloud platform, oracle ,IBM cloud, Rackspace ,Salesforce, Cloud switch, Intacct, Engine yard.

Through this set of services we will rank the cloud services by using clustering and categorization and decision tree algorithms By using the selection process we will select the services and also through selection process only we will rank the services easily. In this dataset we are having the 10 cloud services and regarding that cloud services we are having the data for those services to rank the best one among that services

We will first mainly consider the cost for all the services to rank the best services. The user can easily find out the best service he want. 
Table 1: overall dataset for cloud services

\begin{tabular}{|c|c|c|c|c|}
\hline SNO & Company Name & $\begin{array}{c}\text { Max. } \\
\text { Memory }\end{array}$ & $\begin{array}{c}\text { Max. storage } \\
\text { type }\end{array}$ & $\begin{array}{c}\text { Max. } \\
\text { cost }\end{array}$ \\
\hline 1 & AWS & 256 & 8 & $\$ 3.20$ \\
\hline 2 & MSAZURE & 32 & 8 & $\$ 1.24$ \\
\hline 3 & $\begin{array}{c}\text { Google Cloud } \\
\text { Platform }\end{array}$ & 240 & 6 & $\$ 3.04$ \\
\hline 4 & IBM & 128 & 6 & $\$ 2.23$ \\
\hline 5 & Oracle & 512 & 5 & $\$ 0.15$ \\
\hline 6 & Rackspace & 128 & 7 & $\$ 2.45$ \\
\hline 7 & Salesforce & 0 & 7 & $\$ 3.00$ \\
\hline 8 & Cloud Switch & 0 & 6 & $\$ 2.50$ \\
\hline 9 & Intacct & 0 & 0 & $\$ 1.20$ \\
\hline 10 & Engine yard & 0 & 6 & $\$ 0.40$ \\
\hline
\end{tabular}

\section{a) Cost based clustering}

\begin{tabular}{|c|c|c|c|}
\hline SNO & Company Name & Max. cost & Cluster K \\
\hline 1 & AWS & $\$ 3.20$ & K3 \\
\hline 2 & MSAZURE & $\$ 1.24$ & K1 \\
\hline 3 & Google Cloud Platform & $\$ 3.04$ & K3 \\
\hline 4 & IBM & $\$ 2.23$ & K2 \\
\hline 5 & Oracle & $\$ 0.15$ & K1 \\
\hline 6 & Rackspace & $\$ 2.45$ & $\mathrm{~K} 2$ \\
\hline 7 & Salesforce & $\$ 3.00$ & $\mathrm{~K} 3$ \\
\hline 8 & Cloud Switch & $\$ 2.50$ & $\mathrm{~K} 2$ \\
\hline 9 & Intacct & $\$ 1.20$ & $\mathrm{~K} 1$ \\
\hline 10 & Engine yard & & $\mathrm{K} 1$ \\
\hline
\end{tabular}

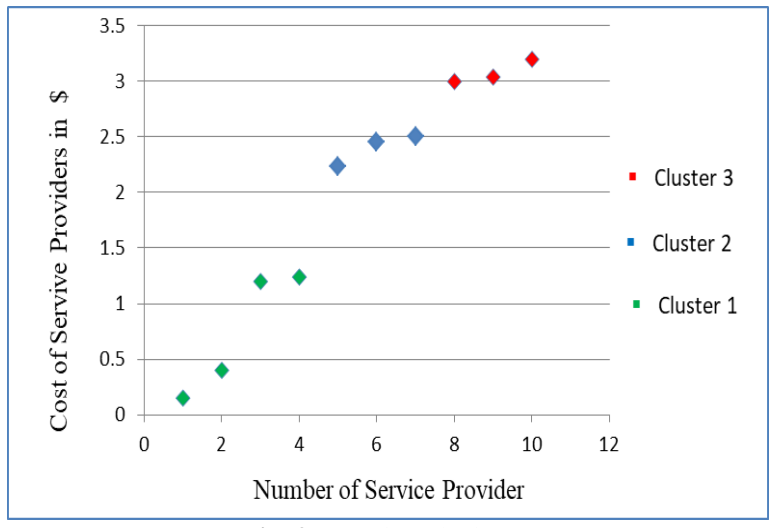

Fig. 3: Cost based Cluster

The output will be like if the user requirement is based on cost means if he need the service provider at $\$ 4$ the we cluster the dataset using the k-means and we categorize the services according to the user requirement if his requirement is $\$ 4$ then we are having the centroid as $\$ 3.5$ means we will categorize that service as the gold his requirement as silver and out of his expectations we will rank it as Bronze. Then we will go for decision tree algorithm through this we can justify the user needs or wish that which is the best service he can choose.

In this process only we will cluster the datasets for all the service providers we will cluster the services in the same process we will rank the services according to the user requirements as above we did for clustering in the same way we will process for all the datasets. We will categorize all the datasets according to the user requirements.

In this process we will first do the clustering mechanism and in clustering mechanism we will cluster all the services of cloud services according to the user requirements and then we will have the user requirement as the centroid value after clustering we will see that which services are near to the centroid value and which are near to the centroid value we will rank that services as the best and we will allot the weightage as gold for best cloud service provider then we will go for decision tree algorithm we will check which services are apt to the user according to that we will prioritize the services and then we will categorize the services according to cluster 1 ,cluster 2 ,cluster 3 .

\section{Conclusion}

In this paper the final conclusion is through clustering we can easily identify the best service provider through the centroid value and it is the easy process to find the best among the services when compared to others. It also takes less time. It is having the ability to perform and maintain with limited downtime. The clustering process will gives the best service provider according to the user requirement. In clustering we will cluster the service which is best for user and later we will perform the categorization process according to the user requirements after categorizing the services we will go for priority decision tree which should have first priority according to the user requirement

In this process we will be having the automated selection process and also by allotting the weightages as (gold, silver, bronze) are used to increase the response time. We can receive the response with in the time limit. finally through clustering we are going to have the best service provider within seconds. The user need not to spend much time for searching the best service provider .By using this clustering process we can save time and energy for searching the best service process.

The best way of finding the best cloud service providers is clustering mechanism. Through this process we can find the services according to the user requirement. The user can request through any parameters which he wants after the request is given it will cluster the parameter through the services which ever services are provided through that we will cluster and rank the best service provider for the parameter which the user has requested

\section{References}

[1] Abassi, R; El Fatmi, S.G, Towards a generic trust management model, in 19th International Conference on Telecommunications, Jounieh pp.1-6 (2012) .

[2] Abawajy, Determining Service Trustworthiness in Intercloud Computing Environments in pervasive Systems, Algorithms, and Networks , 10th International Symposium , pp.784-788(2009).

[3] C. S. Rajarajeswari, M. Aramudhan, (September 2014) Ranking Model for SLA Resource Provisioning Management International Journal of Cloud Applications and Computing, 4(3), 68-80, July-September 2014

[4] Khan K, Malluhi Q (2010) Establishing trust in cloud computing IT Prof 12(5): 20-27. doi:10.1109/MITP.2010.1287.

[5] Li Yang et al, A new Class of Priority-based Weighted Fair Scheduling Algorithm, Physics Procedia ,33 ( 2012 ) 942 - 948.

[6] LIN Guoyuan, WANG Danrul, BIE Yuyul, LEI Min "MTBAC: A Mutual Trust Based Access Control Model in Cloud Computing" china communication, 2014.

[7] M.Aramudhan, M.Saravanan,"Trust Based Ranking Service Models in Cloud Computing Environment".

[8] Michael B, Dinolt G (2010) Establishing trust in cloud computing. IANewsletter 13(2): 4-8. http://iac.dtic.mil/iatac/download/Vol13_No2.pd

[9] Preeti Gulia, Sumedha Sood, "Dynamic Ranking and Selection of Cloud Providers Using Service Level Agreements" International Journal of Computer Applications (0975 - 8887) Volume 72- No.11, May 2013

[10] Preeti Gulia, Sumedha Sood (2013) „Automatic Selection and Ranking of Cloud Providers using Service Level Agreements International Journal of Computer Applications (0975 - 8887) Volume 72- No.11, May 2013.

[11] Qiang Guo, Dawei Sun, Guiran Chang, Lina Sun and Xingwei Wang, Modelling and evaluation of trust in cloud computing environments in International Conference on Advanced Computer Control ,pp.112-116(2011).

[12] Stefan Frey, Claudia L"uthje, Christoph Reich 'Key Performance Indicators for Cloud Computing SLAs': The Fifth International Conference on Emerging Network Intelligence EMERGING 2013 Copyright (c) IARIA, 2013. ISBN:978-1-61208-292-9 
[13] Saurabh Kumar Garg a,*, Steve Versteeg b, Rajkumar Buyyaa „A framework for ranking of cloud computing services ${ }^{\text {ee }}$. Future Generation Computer Systems 29 (2013) 1012-1023 journal homepage: www.elsevier.com/locate/fgcs.

[14] Zhao-xiong Zhou, $\mathrm{He} \mathrm{Xu}$, and Suo-ping Wang, "A Novel Weighted Trust Model based on Cloud," AISS: Advances in Information Science and Service Sciences, Vol. 3, No. 3, pp.115124, April 2011.

[15] T. Padmapriya and V. Saminadan, "Improving Throughput for Downlink Multi user MIMO-LTE Advanced Networks using SINR approximation and Hierarchical CSI feedback", International Journal of Mobile Design Network and InnovationInderscience Publisher, ISSN : 1744-2850 vol. 6, no.1, pp. 14-23, May 2015.

[16] S.V.Manikanthan and K.srividhya "An Android based secure access control using ARM and cloud computing", Published in: Electronics and Communication Systems (ICECS), 2015 2nd International Conference on 26-27 Feb. 2015,Publisher: IEEE,DOI: 10.1109/ECS.2015.7124833. 